\title{
MENSTRUAL CYCLE CONTROL - A CONTROVERSIAL EXAMPLE OF HUMAN ENHANCEMENT IN RELATION TO WOMEN'S BODY AND PSYCHE
}

\author{
Lucia Martinelli ${ }^{1}$, Srećko Gajović ${ }^{2} \&$ Jiwon Shim $^{3}$ \\ ${ }^{1}$ MUSE - Science Museum, Trento, Italy \\ ${ }^{2}$ Croatian Institute for Brain Research, University of Zagreb School of Medicine, Zagreb, Croatia \\ ${ }^{3}$ Department of Philosophy, Dongguk University, Seoul, Korea
}

received: 29.6.2020;

revised: 1.10.2020;

accepted: 9.10 .2020

\begin{abstract}
SUMMARY
By discussing the position of bio-conservatism and transhumanism, we question if the women menstrual cycle control would represent a new way toward a more responsive relation with one's own physical and mental health, a choice of freedom from undesired physiological conditions, a medicalization of a natural physiological event or an innovative carrier of social stigma against the women. We argue that the advancement of medical science may allow women a choice if to regulate own menstrual cycle, offering them as well a right to intervene responsibly on their own body and psyche. Accordingly, a post-human society could provide a suitable coexistence between women who claim menstruation as the biological essence and those who claim it as an option.
\end{abstract}

Key words: menstrual cycle control - mental health - psyche - bio-conservativism - transhumanism

$* * * * *$

\section{INTRODUCTION}

On not only curing diseases and preventing illness, the attention of nowadays medicine is also focused on assuring physical and psychical well-being (WHO 1946). According to this framework, the technological expertise to control human body physiology is one of the most challenging future opportunities offered by biomedical advancements. Remarkable results have been obtained in the field of reproduction, where the medical tools were developed with abilities for regulating the "natural" course of human biology. The interventions on the female reproductive physiology to prevent or to post-pone pregnancies as well to circumvent sterility are excellent examples of biology innovations in the field. However, these advances proved to be carriers of ambivalent impacts on personal and social choices. The interventions in the human reproduction are as well a source of enduring controversies between medical necessity and elective reasons (Martinelli et al. 2015).

In this paper, we discuss the case of controlling the women menstrual cycle and, in particular the menstrual bleeding, for non-medical reasons. We do not focus on the potential health risks associated with the practice of assumed hormonal treatments. Rather, by discussing the positions of bio-conservatism and transhumanism, we question if this strategy would represent a new way toward a more responsive relation with one's own body and psyche.

\section{THE MENSTRUATION AT THE BOUNDARY BETWEEN WOMAN PHYSIOLOGY AND SOCIAL STIGMA}

Menstrual cycle represents a relevant biological, intimate and socio-cultural experience for females during a quite long period of their lifetime. It is estimated that over 800 million women menstruate daily. Considering the first (12-15 years of age) and the last (45-55 years of age) appearance of the menstruation, its frequency ( 21 to 31 days) and duration ( 2 to 7 days), it has been calculated that women have an average of 450-500 menstruation cycles and an average of 2,500 bleeding days. These data vary among industrialized and developing countries, according to health and life quality status, number of births and attitude toward breastfeeding. Primary dysmenorrhea, characterized by painful spasmodic cramping in the lower abdomen in the absence of pelvic pathology, may occur as the most common gynecological condition among women of reproductive age, ranging from $45-95 \%$ of menstruating women (Iacovides et al. 2015). Premenstrual disorder (PMS) or the more severe (and less common) premenstrual dysphoric disorder (PMDD) can affect women through somatic symptoms (head, joint and muscular pain; breast tenderness or swelling; weight gain) or psychiatric symptoms (change in mood; anxiety; social withdrawal) (Hofmeister \& Bodden 2015). Symptoms have been classified as somatic (head, joint and muscular pain; breast tenderness or swelling; weight gain) or as psychiatric (change in mood; 
anxiety; social withdrawal) and their persistence and severity tend to fluctuate. Cyclical changes in estrogen and progesterone levels seem to be the cause of these symptoms.

Historically and culturally, a woman is defined as healthy if she has her period regularly. Some pathologies, in fact, have as a symptom the alteration of menstrual rhythmicity, including absence of menstruation (amenorrhea), thus deserving to be treated. Different cultures view menstrual bleeding in different ways assumed over the centuries on a cultural and anthropological level, which, however, do not have scientific basis. These include a number of contradictory values (purifying significance: keeping the woman healthy in itself, renewing the body; sacral meaning: motherhood) and distastes (impurity emblem: making the woman unclean or contaminant; detrimental feminine: hysteria). The taboo and social stigma against menstruation seem to be prevalent in every culture and religion of the past and the present, reflecting somatophobia and misogyny (Johnston-Robledo \& Chrisler 2013).

Because of the prejudiced belief that a menstruating woman should avoid some activities, menstruation keeps women from their daily duties, thus masking a form of women's segregation. Stigma has been noticeably analyzed and described in literature, the feeling of embarrassment, being a target of an adverse projection coming from "the others"; the jargon as well as the silence about it; the women's narratives (recently also shared through on-line spaces); the communication used in the advertisements for sanitary menstrual products. In their analysis, Johnston-Robledo \& Chrisler (2013) point out the fact that "if people hold cultural believes that the menstrual cycle causes women to be physically (menstrual phase) or mentally (premenstrual phase) disordered, then the stigma of menstruation also marks women as unfeminine, ill, disabled, out-of-control, or even crazy". As an outcome, such marks would produce negative consequences for women's sexuality, physical and mental health, wellbeing and social status.

It is hopeful that the recent arguments in favor of a "menstrual justice" coming from the medical society as well as from the feminist, cultural and counter-culture movements would help raise consciousness and fighting against such negative stigmatization. The Society for Menstrual Cycle Research [http://www.menstruation research.org/] aims "to be the source of guidance, expertise, and ethical considerations for those interested in the menstrual cycle". The Red Web Foundation [https://www.theredweb.org/] wants "to connect and inform people about the menstrual cycle through education, awareness, and activism". The \#HappyToBleed Facebook and Twitter campaign started by the Indian college student Nikita Azad to protest against religious stigmata states "It may be painful, but it's perfectly normal to bleed and it does not make me impure". The audacious artists Vanessa Tiegs [https://www.vanessatiegs.com/menstrala/] and
Petra Paul [http://mum.org/armenpau.htm] exploit their menstrual blood as colors for their provocative-shocking artistic paintings. The outstanding exhibition "Periods A brief history", programmed to run during 2020 at the Vagina Museum of London, specifically aims at highlighting how periods are perceived and how a lack of understanding has affected (and still do affect) people's lives [https://www.vaginamuseum.co.uk/exhibitions/futureexhi bition].

\section{FROM A NORMATIVE BIOLOGICAL STATUS TO AN ELECTIVE PHYSIOLOGICAL OCCURRENCE}

Menstrual cycle control proved to be an important medical achievement rising personal and social value for life quality enhancement. Since the 1960s the unwanted pregnancy prevention offered by hormonal treatment is a milestone for the woman emancipation process, allowing a more active and responsible control on motherhood with the separation of the reproductive act from the sexual act. This latter becomes even more explicit in the case of assisted reproductive techniques (ARTs) where fertilization may occur outside of human body. Moreover, ARTs may generate a remarkable shift from privacy/intimacy to social/collective, like in a case of heterologous and surrogate reproduction where "becoming a parent" (both mother and father) involves a number of actors, revising the traditional meaning of family and kinship. From the ethical viewpoint, both contraceptive devices and medical conception techniques may be regarded as useful and "permissible" tools, at least for therapeutic reasons. Medical purposes, in fact, recommend avoiding pregnancy during aggressive therapeutic treatments (such as chemotherapy and radiotherapy, for instance), whilst, in various countries, sterility is considered a pathological condition and therefore endorsing the use of ARTs. However, when applied for non-medical reasons, the same practices still raise questions about their technological, ethical, sociological and legal aspects (Martinelli et al. 2015).

Similarly, when associated with a series of disturbing symptoms often resulting in acknowledged pathology (e.g. physical pain and mood swings that can be debilitating), menstrual cycle control is medically advised. It is for instance prescribed in the case of endometriosis symptoms, which are aggravated by menstruation (Blanchard 2003). Hormone-releasing intrauterine devices (IUD), usually lasting for 5 years and used as effective contraceptives (such as for instance Mirena), became recently popular to avoid menstrual bleeding (Dhamangaonkar et al. 2015). The cycle suppression is also obtained with daily progesterone-only pills (POPs), the 3-monthly progestinonly injection of Medroxyprogesterone acetate (DMPA) or the progestin-based subdermal implant that is viable for at least 3 years. In comparison to the traditional 
oral contraceptives, Mirena offers the advantage to avoid a regular administration of pills in repeated cycles and effective control of menstrual bleeding (shorter duration and amount), good tolerability and a favorable cost-benefit ratio.

As for non-medical reasons, suppression of menstrual bleeding is applied in extreme situations where practicalities of personal hygiene are a constraint, like during spaceflights for female astronauts (Jain \& Wotring 2016) and under austere situations, like in a military environment, in particular during combats (Powell-Dunford et al. 2011). According to various interviews available on the Internet, menstrual cycle can be a problem for women who practice sports at high levels. However, some try to regulate it and others, who do not consider it a problem, cope with it without consequences even during important competitions. Apart from those specific reasons, there is a rising opinion that being without inconvenient bleeding of menstruation improves the quality of life and even that bleeding may be not only inconvenient, but harmful for the women's physical and mental health. Today menstrual bleeding is not any more necessary as a signal that a pregnancy did not occur. Finally, economic rationale has been advocated against menstruation, estimating the costs of sick leave from work and of the hygiene devices needed (to both produce and dispose) during the bleeding phases (Blanchard 2003).

\section{MENSTRUAL CYCLE CONTROL AND HUMAN ENHANCEMENT}

In the classical meaning, the goal of medicine is to alleviate pain and treat disease. Because of the medical technology development, however, the medical intervention could be used not only for medical but also for elective reasons, thus not only for recovering unhealthy body and restoring peace of mind (i.e. for "patients") but also for enhancing healthy body and psychological equilibrium (i.e. for "customers"). As previously defined (Parens 1998), "The term enhancement is usually used in bioethics to characterize interventions designed to improve human form or functioning beyond what is necessary to sustain or restore good health". Worth stressing, according to Juengst \& Moseley (2019), "There are no "enhancement technologies" per se. Whether a given biomedical intervention counts as an enhancement depends on how it is used". Accordingly, emblematic examples of human enhancement are: the plastic surgery to improve one's appearance; the doping to increase physical fitness and athletic capability; the drugs to modulate emotions and mood (Fluoxetine, sold under the brand names Prozac among others), concentration capability (Methylphenidate, sold under the trade name Ritalin among others), as well as to strengthen sexual ability (Sildenafil, sold under the brand name Viagra among others)
When discussing human enhancement, three central issues to be considered are the "goal" of medical intervention; the "method and means" of the intervention; and the "violation" of "human nature" (Shim 2012). The "goal" of medical intervention relates to the general purpose of medicine as a science and practice. Whenever used for medical necessity, the medical intervention is considered appropriate. When its goal is human enhancement, on the contrary, it becomes controversial. This would be the case, for instance, of breast removal surgery in breast oncological patients versus female athletes to improve sport records. The "method and means" of intervention relate to the controversy between traditional/conventional/"natural" and "not-natural". This is the case of drinking coffee versus assuming drugs to stimulate concentration and wake, as well as controlling reproduction with "natural" versus medical devices. The "violation" of "human nature" for the purpose of enhancement is the most controversial issue since such interventions would deeply operate on humans' typical biological/physical characteristics. This would be the case of transforming a 10-finger-hand into a 12-finger-hand to enhance the capability of playing piano.

There are different positions about the human modification through medical intervention, the extreme opposition being represented by bio-conservatism and transhumanism. Leon Kass (2003), the representative of the bio-conservatives, considering that human nature is unique to humans, takes a negative position on transforming human nature through medical intervention: "Most of the given bestowals of nature have their given species-specified natures: they are each and all of a given sort. Cockroaches and humans are equally bestowed but differently natured. To turn a man into a cockroach - as we don't need Kafka to show us - would be dehumanizing. To try to turn a man into more than a man might be so as well. We need more than generalized appreciation for nature's gifts. We need a particular regard and respect for the special gift that is our own nature (and, be the way, also that of each of our fellow creatures)".

In contrast, transhumanists not only imagine more advanced humans, they also consider feasible and positive the technical intervention to overcome human limitations. Nick Bostrom (2003) accordingly, states: "We can conceive of aesthetic and contemplative pleasures whose blissfulness vastly exceeds what any human being has yet experienced. We can imagine beings that reach a much greater level of personal development and maturity than current human beings do, because they have the opportunity to live for hundreds or thousands of years with full bodily and psychic vigor. We can conceive of beings that are much smarter than us, that can read books in seconds, that are much more brilliant philosophers than we are, that can create artworks, which, even if we could understand them only on the most superficial level, 
would strike us as wonderful masterpieces. We can imagine love that is stronger, purer and more secure than any human being has yet harbored." Transhumanism is a way of thinking about the future that is based on the premise that the human species in its current form does not represent the end of our development but rather a comparatively early phase. Transhumanists place a high value on autonomy: the ability and right of individuals to plan and choose their own lives (Bostrom 2003). Whilst bio-conservativism would oppose the control of menstrual cycles to improve women's life quality (human enhancement), transhumanism would be in favor of this practice. According to transhumanism, in fact, humans are obliged to improve their physical and mental states. Worth remembering the provocative "Listening to Prozac" best seller popular science book, where the psychiatrist Peter Kramer (1994) discusses how the use of the antidepressant drug Prozac can go beyond just alleviating negative emotions by producing personality changes in a sort of "cosmetic pharmacology" enabling psychopharmaceuticals to be used in "self-creation" projects.

As controlling women menstrual cycle has the aspect of improving women functioning "beyond what is necessary to sustain or restore good health", it can be classified as human enhancement. Accordingly, as a specific form of human enhancement, it can be analyzed in the light of the three challenges above considered. As for "goal", menstrual cycle control used primarily to improve life quality instead of for medical or contraception reasons would not fit in the purpose of medicine in the traditional sense. However, health is not any more considered as a pure absence of illness, but "a state of complete physical, mental and social well-being" (WHO 1946). Nowadays medicine is certainly oriented toward achieving health, and pursue prevention, hence the boundary between treatment and enhancement, i.e. to cure disease versus to assist well-being, is becoming more and more ambiguous. As for "method and means", the use of invasive methods such as the intrauterine devices that are inserted into the body (Mirena, for instance) would be differently evaluated if used for (necessary) medical care versus (elective) life quality improvement. As for the "violation" of "human nature", controlling menstrual cycle through medical intervention may be viewed as a violation of the human (female) nature or, quite opposite, may be an enhancement of the distinctive feminine features. In relation to menstrual cycle control, the biomedical science opens the doors to new perspectives concerning the relationship between given female body and choosing female body. In offering this, it also raises questions about what a woman's biological essence is and implies that the human biological essence is not fixed, but in progress, as well.

\section{TO BLEED OR NOT TO BLEED: A POLEMIC CHOICE}

A number of surveys conducted across Europe, North America and Latin America to assess attitudes towards monthly bleeding found that a significant majority of women would prefer to avoid the unpleasant aspects of menstruation and its negative impact on private and professional lives (Szarewski et al. 2012). Critical voices, on the other hand, raise questions about threats of such vision. The ambiguous relation between the concept of healthy and unhealthy status can be distorted, giving an impression that a "natural" physiological occurrence of menstrual cycle can be considered an unhealthy or even pathological condition. Just opposite to that, a situation of wellbeing without menstrual cycle due to the pharmacological treatment could be considered not only desirable, but as well healthy. This medicalization of the menstrual cycle may produce in healthy women the distressing perception to be ill, disordered and "out of place". In addition, it may reinforce, in the social imaginary, the unfounded mystifications about the menstrual cycle as a distasteful occurrence. Thus, in this view, a medical innovation becomes a novel carrier of enduring bias against the women's body and psyche.

\section{CONCLUSIONS}

In conclusion, the advancement of medical science allows the women already today to choose to adopt or not adopt the innovation, providing them the tools and the rights to responsibly regulate their own body and psyche. Accordingly, a post-human society would be a society where it would be suitable the coexistence between (women) people who claim menstruation as the biological essence and (women) people who claim it as an option.

\section{Acknowledgements:}

This work was supported by the Ministry of Education of the Republic of Korea and the National Research Foundation of Korea (NRF-2017S1A5A8021687)

Conflict of interest: None to declare.

\section{Contribution of individual authors:}

Lucia Martinelli: writing of first draft, editing of draft, approval of the final version.

Srećko Gajović: editing of draft, final revision and approval.

Jiwon Shim: writing of first draft, approval of the final version. 


\section{References}

1. Blanchard K: Life without menstruation. The Obstetrician \& Gynaecologist 2003; 5:134-7

2. Bostrom N: Human genetic enhancements: a transhumanist perspective. J Value Inq 2003; 37:493-506

3. Dhamangaonkar PC, Anuradha K, Saxena A: Levonorgestrel intrauterine system (Mirena): An emerging tool for conservative treatment of abnormal uterine bleeding. $J$ Midlife Health 2015; 6:26

4. Hofmeister $S$, Bodden S: Premenstrual syndrome and premenstrual dysphoric disorder. Am Fam Physician 2016; 94:236-40

5. Iacovides S, Avidon I, Baker FC: What we know about primary dysmenorrhea today: a critical review. Hum Reprod Update 2015; 21:762-78

6. Jain V, Wotring VE: Medically induced amenorrhea in female astronauts. npj Microgravity 2016; 2:16008

7. Johnston-Robledo I, Chrisler JC: The menstrual mark: Menstruation as social stigma. Sex Roles 2013; 68:9-18

8. Juengst E, Moseley D: Human Enhancement. In: Edward N. Zalta, eds. The Stanford Encyclopedia of Philosophy (Summer 2019 Edition). https://plato.stanford.edu/entries/ enhancement/ (accessed 2020 June 29)

9. Kass LR: Ageless bodies, happy souls: biotechnology and the pursuit of perfection. New Atl 2003; 9-28

10. Kramer PD, Brody EB: Listening to Prozac: A psychiatrist explores antidepressant drugs and the remaking of the self. J Nerv Ment Dis 1994; 182:362

11. Martinelli L, Busatta L, Galvagni L, Piciocchi C: Social egg freezing: a reproductive chance or smoke and mirrors? Croat Med J 2015; 56
12. Parens E: Enhancing human traits: Ethical and social implications. Georgetown University Press, 1998

13. Paul Petra: The Art of Menstruation at the Museum of Menstruation and Women's Health.

http://mum.org/armenpau.htm (accessed 2020 June 29)

14. Powell-Dunford NC, Cuda AS, Moore JL et al.: Menstrual suppression for combat operations: advantages of oral contraceptive pills. Women's Heal Issues 2011; 21:86-91

15. Shim J: „Enhancement" Die Legitimität von Eingriffen am gesunden menschlichen Körper, PhD diss., Westfälische Wilhelms-Universität Münster, 2012

16. Society for Menstrual Cycle Research. www.menstruationresearch.org (accessed 2020 June 29)

17. Szarewski A, von Stenglin A, Rybowski S: Women's attitudes towards monthly bleeding: results of a global population-based survey. Eur J Contracept Reprod Heal Care 2012; 17:270-83

18. The Red Web Foundation: www.theredweb.org (accessed 2020 Apr 15)

19. Vanessa Tiegs: Menstrala. www.vanessatiegs.com/menstrala (accessed 2020 June 29)

20. Vagina Museum. Future Exhibition: Periods: A brief history. www.vaginamuseum.co.uk/exhibitions/futureexhibition (accessed 2020 June 29)

21. WHO: Preamble to the Constitution of WHO as adopted by the International Health Conference, New York, 19 June 22 July 1946; signed on 22 July 1946 by the representatives of 61 States (Official Records of WHO, no. 2, p. 100) and entered into force on 7 April 1948. The definition has not been amended since 1948

Correspondence:

Jiwon Shim, PhD

Department of Philosophy, Dongguk University

\#828, 310 Hall, 84 Heukseok-ro, Dongjak-gu, Seoul, 06974, Korea

E-mail:g1dmp@daum.net 